\title{
WIP: Curricular Renewal for System Engineering: Project-based Capstone Framework to Hatch Autonomy and Creativity
}

\section{Dr. Chao-Yang Cheng, National Chiao Tung University}

Chao-Yang Cheng is a postdoctoral researcher from the Institute of Electrical and Control Engineering of National Chiao Tung University in Taiwan. He majored in educational psychology and minor in multilevel linear models. Flow theory, daily reconstruction method, classroom experience, immediate process feedback module, capstone teaching and learning, and engineering education are central to his area of study.

\section{Prof. Yu-Lun Huang, National Chiao Tung University}

Yu-Lun Huang received the B.S. and Ph.D. degrees in computer science and information engineering from the National Chiao-Tung University, Taiwan, in 1995 and 2001, respectively. Currently, she is Associate Dean of Office of Academic Affairs and Associate Professor in Department of Electrical and Control Engineering, National Chiao-Tung University, Taiwan. Her research interests include system engineering education, wireless security, secure testbed design, embedded software, embedded operating systems, network security, secure payment systems, VoIP, and QoS.

\section{Prof. Bing-Fei Wu, National Chiao Tung University \\ Prof. Yon-Ping Chen, National Chiao Tung university}

Yon-Ping Chen received the B.S. degree in electrical engineering from National Taiwan University, Taiwan, in 1981, and the M.S. and Ph.D. degrees in electrical engineering from the University of Texas at Arlington, USA, in 1986 and 1989, respectively. He is currently a Distinguished Professor with the Department of Electrical Engineering, National Chiao Tung University, Taiwan. His researches include control, image signal processing, and intelligent system design.

\section{Prof. Sunny S. J. Lin, National Chiao Tung University}

Dr. Sunny S. J. Lin is Educational Psychology Professor at National Chiao Tung University, Taiwan. Her first research interest is in applying cognitive theories of learning and motivation to derive design principles of e-learning function and multimedia program using self-report, response pattern analysis, experience sampling and eye tracking techniques, for which she received outstanding research award from National Science Council Taiwan in 2005. Her second research interest is in exploring longitudinal effects of problematic Internet use on adolescent wellbeing involving diversified undergraduates and high school students. 


\title{
WIP: Curricular Renewal for System Engineering: Project-based Capstone Framework to Hatch Autonomy and Creativity
}

\author{
Introduction
}

This study describes a renewed System Engineering (SE) curricular structure and pedagogical principles; it provides evidence of student learning experiences and outcome qualities to examine whether the goals of curricular renewal have been achieved. Figure 1 illustrates the curricular framework, pedagogical process, student learning process, and evaluation technique. The curriculum renewal described in this study is part of a capstone reorganization for the Electronic Engineering Department (EE), which has categorized undergraduate-level courses into the cornerstone, keystone, and capstone. Professors from SE and Educational Psychology formed a cross-disciplinary team which decided that the renewal process abides by the "Progress Principle" [1]. The Progress Principle stresses on the shaping of a positive study life. Not only does it make students feel happy and engaged in their work, but it also leads to better performance including higher creativity and productivity. A great study life is a continuous stream of emotions, favorable perceptions of the class, classwork, and their teachers/peers, and positive motivations that students experience throughout class days. The goals of students learning were expected to be "autonomy" (demonstrated from active engagement in productive activities) and "creativity" (shown from the creative level of project outcomes).

Three courses were selected and integrated into the core of Control Systems (depicted in the left column of Figure 1), which is among one of the six centers of the EE department in the National Chiao Tung University. The serial courses specifically focus on innovative engineering applications of the 4Cs (Computer, Communication, Customer, and Car Electronics), with "Automatic Control Systems and Lab" addressing microcontroller-based applications, "Dynamic Control Systems" addressing personal computer-based applications and micro-controller, and "Embedded Operating Systems" addressing embedded systems and ARM-based applications. To promote the success of the capstone renewal, two alignment pedagogy strategies, project-based learning and creative-design facilitation, have been adopted. The instructional modules were then redesigned to keep them constant across the three courses but with various weightage among modules according to the level in the capstone framework. The modules included: (I) Announcing and reemphasizing the new learning goal; (II) Lectures on SE, Physics, and Mathematical models; (III) Small-scale classwork including innovative ideas developing through mind-mapping or SCAMPER, group-function practice, math problem-solving, individual and collaborative experiments/labs of SE, and quizzes; (IIII) Mid-term and final examinations containing proportions of alternative and traditional formats; and (IV) A group project aiming to integrate concepts and skills of SE with an ill-structured format to allow innovation.

During a semester, there are four cyclical processes of project-based learning (depicted in the central column of Fig. 1): (1) Lecture guidance from teacher; (2) Students' inquiry with support from teacher and TAs; (3) Students' interpretation of inquiry results; and (4) Actions in conducting projects. Additionally, creative thinking 
and product design were also plotted into four instructional steps: (A) Activating prior knowledge; (B) Practices of creative ideas; (C) Selection of the proper one from multiple creative actions; and (D) Task review to confirm creativity achievement. Finally, a final presentation has been held for a panel of experts to evaluate project outcomes.

The outcome evaluation of curricular renewal is manageable, but the process of evaluation is often very challenging. Process evaluation is commonly conducted by techniques such as observation, interview, or case study that are often criticized for using a small sample size and may result in findings that lack the ability to be generalized for a wider context. Furthermore, it is difficult to find comparison or a control group for focal participants in the observation, interview or case study thus making the data interpretation subjective. This study provides an innovative investigation strategy, Day Reconstruction Method (DRM), to conduct process evaluation for curricular and instructional innovation.

The first purpose of this study aims to use the latent profile analysis (LPA) to explore SE and non-SE students' experiences in daily activities. A repeated investigation proved that the Day Reconstruction Method (DRM) is adapted to collect students' experiences. The study uses the LPA to determine the optimal group numbers and cluster the variables of experiences and frequency in each daily activity to form homogeneous groups. Then, this study compared the groups to figure out which group was more willing to plan and manage their daily schedules in productive activities (e.g. studying in school and after school). Second, a panel of SE experts reviewed collaborative products of the final project according to the Consensual Assessment Technique (CAT). The authors conducted a case study to compare creative performance in final products among the three SE courses.

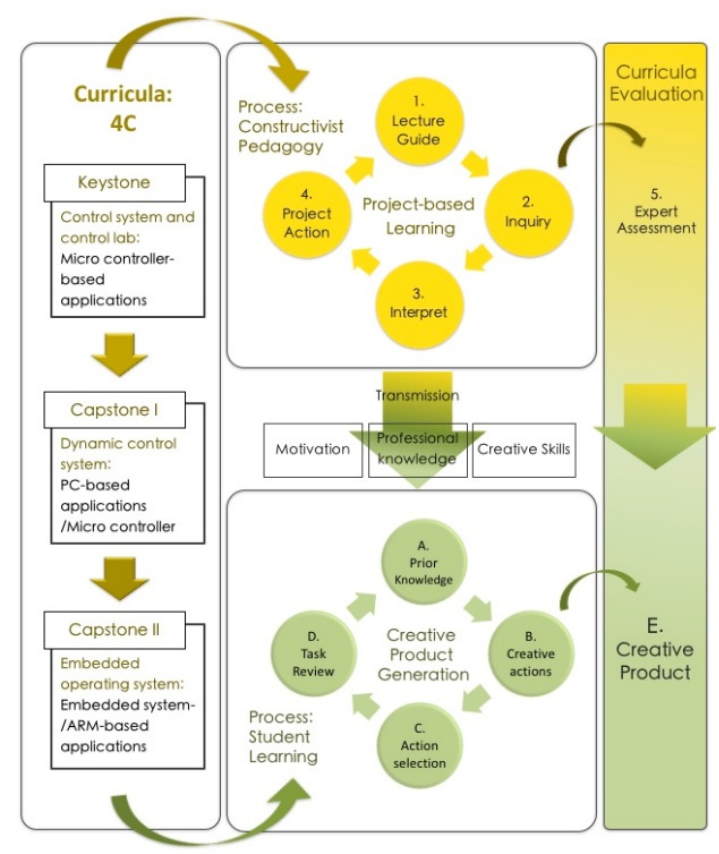

Figure 1. Framework of System Engineering Curricula Renewal Structure, Pedagogical Process, and Outcome Evaluation 
The research questions are as follows:

RQ1: In a group of college students, can SE students be differentiated from others based on their learning experiences in three SE courses through a statistical clustering technique (Latent Profile Analysis, LPA)?

RQ2: When the project outcomes were evaluated using the Consensual Assessment Technique, to what creative levels are the outcomes reached? Are there differences regrading student creativity among the three SE courses?

Method

Participants.

The data of this study were collected from five universities in Taiwan. A total of 271 effective samples were collected. There were 40 SE students (15\%) and 231 from other classes, which consisted of 101 males (38\%) and 161 females $(59 \%)$. The average age of the students was 20.12 years old $(S D=1.74)$.

\section{Procedure.}

DRM: The Day Reconstruction Method (DRM) [2] was used to collect repeated data to measure activity-specific experiences of the preceding day, for all activities which occurred in that day. A total of 271 college students reported 5,258 episodes by the DRM. Experiences were from self-report with 10 dimensions: concentration, time perception, satisfaction, enjoyment, balance of challenge and skill, with clear goals, task importance, voluntary, focusing on task, anxiety, tiredness during six types of college activities: class, work, social, leisure (passive and active), and maintenance. The frequencies of activities an individual participated in were recorded. Autonomy is defined as positive experiences and high engagement in productive activities.

LPA: We adopted a person-centered approach, the Latent Profile Analysis (LPA). LPA is a statistical method that takes the configuration of multiple variables into consideration so that researchers can identify groups that differ quantitatively at the profile level, qualitatively in the profile shape, or both [3].

CAT: The creativity levels of project products were evaluated using the Consensual Assessment Technique (CAT) [1] by 10 SE experts. The scoring rubric included seven items on a ten-point scale to evaluate the products' originality, professional quality, and elaboration. The interrater reliability was tested by Kendall's W, and it was acceptable (Kendall's $\mathrm{W}=0.384, p<0.001$ ). 
The first goal was to classify 271 college students (including 40 SE students) through their learning experiences and frequencies of activities to examine how the profiles were related to autonomy. The LPA statistics could suggest multiple clustering solutions, and the probabilities of each solution could be analyzed so that the researcher can make a selection. The results from the LPA suggest that college students presented three profiles with different magnitudes in the clustering variables. The model fit statistics for the LPA are displayed in Table 1. The solution with three profiles is selected as the best-fit model.

Table 1. Latent Profile Fit Indices for Different Group Solutions

\begin{tabular}{|c|c|c|c|c|c|c|c|c|}
\hline \multirow{2}{*}{$\begin{array}{l}\# \\
\text { Groups }\end{array}$} & \multirow[t]{2}{*}{$\mathrm{BIC}$} & \multirow[t]{2}{*}{ ABIC } & \multirow[t]{2}{*}{$p$-VLMR } & \multirow[t]{2}{*}{ Entropy } & \multicolumn{4}{|c|}{ Group Size \% } \\
\hline & & & & & $\mathrm{G}_{1}$ & $\mathrm{G}_{2}$ & $\mathrm{G}_{3}$ & $\mathrm{G}_{4}$ \\
\hline 1 & 13247.196 & 13139.392 & & & 100 & & & \\
\hline 2 & 12441.920 & 12223.142 & 0.000 & 0.997 & 84.87 & 15.12 & & \\
\hline 3 (best fit) & 12018.505 & 11688.752 & 0.0155 & 0.950 & 54.61 & 29.88 & 15.49 & \\
\hline 4 & 12013.761 & 11573.033 & 0.7681 & 0.954 & 51.66 & 29.15 & 15.49 & 0.03 \\
\hline
\end{tabular}

The best-fit model shows there are three distinct profiles which could be labeled as the (1) median group, (2) staying in comfort-zone group, and (3) autonomy group. In general, the median group had the largest number of students ( $\mathrm{n}=148$; Fig. 2$)$, and the clustering variables in this group were moderate. Majority of the clustering variables (e.g. concentration, satisfaction, clear goal) in the staying in comfort-zone group ( $\mathrm{n}=81$; Fig. 2 ) were higher than the other groups. However, the students in the staying in comfort-zone group perceived the lowest enjoyment and the highest tiredness and anxiety than those in other groups. The students in the autonomy group $(\mathrm{n}=42$; Fig. 2) presented the right balance between challenges and skills. They also had higher enjoyment, focusing on the task, and voluntary, with more frequent involvements in learning and productive activities than the other groups. The autonomy students reported less anxiety, tiredness, and involvement in maintenance activities as well.

The most interesting result is that all the SE students were classified into the autonomy group. Moreover, the ANOVA results showed they perceived class time significantly shorter (59.24 minutes; median group: 93.90 minutes; staying in comfort-zone group: 99.88 minutes) and reported to have more activities in a course. The evidence showed that the students in the autonomy group could perceive and recognize the transfers among different subjects of courses and were also eager to participate in productive activities (e.g. the participation in project-based learning and after-school learning). 


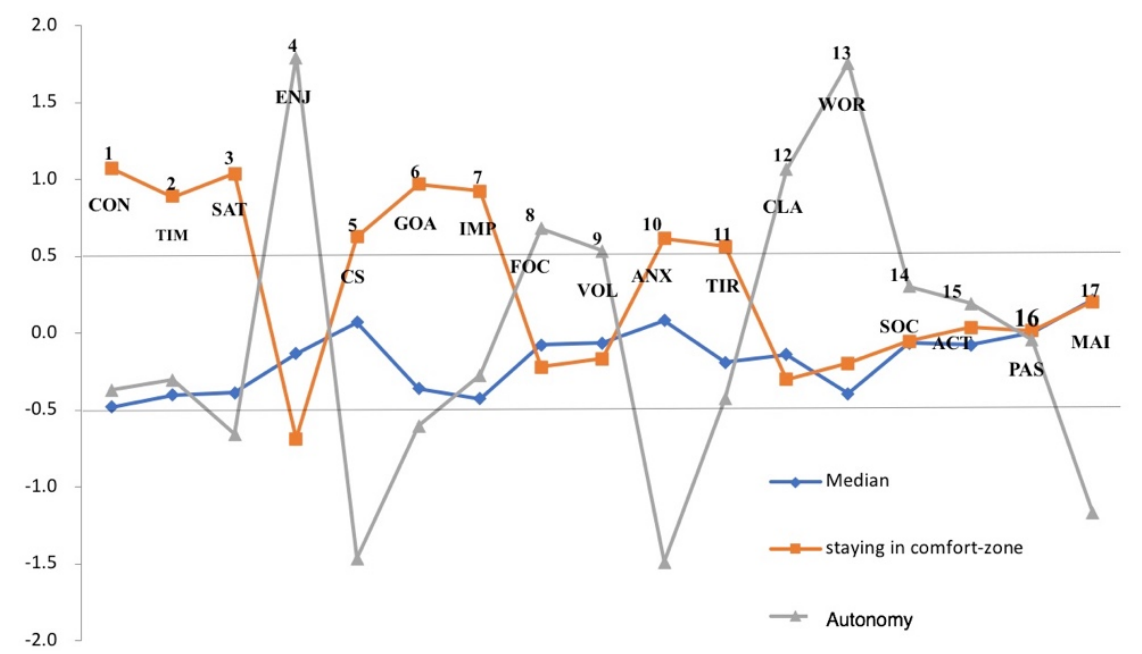

Figure 2: The Latent Profiles Based on Optimal Experiences and Frequency in Daily Activities Note. $\mathrm{CON}=$ concertation, $\mathrm{TIM}=$ time perception, $\mathrm{SAT}=$ satisfaction, $\mathrm{ENJ}=$ enjoyment, $\mathrm{CS}=$ balance of challenge and skill, $\mathrm{GOA}=$ with clear goal, $\mathrm{IMP}=$ task importance, $\mathrm{FOC}=$ focus on task, $\mathrm{VOL}=$ voluntary, $\mathrm{ANX}=$ anxiety, $\mathrm{TIR}=$ tiredness, $\mathrm{CLA}=$ class, $\mathrm{WOR}=$ work, $\mathrm{SOC}=$ social, $\mathrm{ACT}=$ active leisure, $\mathrm{PAS}=$ passive leisure, $\mathrm{MAI}=$ maintenance .

Experts rated the levels of creativity for all projects' products, and the results showed that most products were good in the dimension of professional quality and lower in dimensions of creativity (originality and elaboration). The products designed in the course "Embedded Operating Systems", which is in the highest level of capstone framework, were evaluated as being the highest in the level of creativity. It is reasonable when students have obtained more professional knowledge and aim to design products similar to those in a real high-tech market, the authentic creativity could be observed. Projects produced from lower-level classes (Automatic Control Systems and Lab and Dynamic Control Systems) are high in technical resolution but lack calibration to real-world application.

\section{Discussion}

A curricular renewal for the capstone should be integrated into the three System Engineering (SE) courses (Automatic Control Systems and Lab, Dynamic Control Systems, and Embedded Operating Systems) to encourage project-based learning and creative thinking in the Department of Electrical Engineering at the National Chiao Tung University of Taiwan. This study provides evidence of student learning experiences and project outcome qualities to examine whether the goals of curricular renewal have been achieved. Students from the three SE courses reported to have higher positive experiences (e.g. concentration, enjoyment) and lower negative experiences (e.g. tiredness) and were grouped by the LPA statistical analysis as the autonomy group, which is significantly different from the others (median group and staying in comfort-zone group). We suggest that the curriculum renewal was successful and can be generalized to other courses in the EE Department if teachers are devoted and TAs are trained properly in assisting the project-based capstone course. 


\section{Acknowledgement}

The authors would like to thank the anonymous referees for their valuable and constructive comments. This research is supported by Ministry of Science and Technology, Taiwan, under Grant MOST 105-2511-S-009 -019 -MY3 and NSC 1022511-S-009 -015 -MY3.

\section{References}

[1] T. Amabile and S. Kramer, The Progress Principle: Using Small Wins to Ignite Joy, Engagement, and Creativity at Work. New York, NY: Harvard Business Press, 2011.

[2] D. Kahneman, A. B. Krueger, D. A. Schkade, N. Schwarz, and A. A. Stone, “A survey method for characterizing daily life experience: The day reconstruction method," Science, vol. 306, no. 5702, pp. 1776-1780, 2004.

[3] H. W. Marsh, O. Lüdtke, U. Trautwein, and A. J. Morin, "Classical latent profile analysis of academic self-concept dimensions: Synergy of person-and variable-centered approaches to theoretical models of self-concept," Structural Equation Modeling, vol. 16, no. 2, pp. 191-225, 2009. 